\title{
Gastric- and intestinal-type IPMN: two of a kind?
}

\author{
Michael Noë ${ }^{1,2}$ (D) Lodewijk A. A. Brosens ${ }^{2}$ (D) \\ Received: 14 April 2020 / Revised: 14 April 2020 / Accepted: 22 April 2020 / Published online: 12 May 2020 \\ (C) Springer-Verlag GmbH Germany, part of Springer Nature 2020
}

Intraductal papillary mucinous neoplasms (IPMNs) are precursor lesions to pancreatic cancer. They consist of cystically dilated ducts that are clinically detectable by imaging and are lined by neoplastic epithelium. Their detectability, relative frequent occurrence in the population, and risk for progression into pancreatic cancer, a disease with a dismal prognosis, have resulted in a clinical need for a better understanding of these lesions. In order not to overtreat patients, risk stratification of IPMN and either conservative management or prophylactic treatment is considered the preferable strategy.

IPMNs can be classified into different subtypes, based on the expression of specific mucins and the morphological resemblance to the epithelium of other gastrointestinal organs: gastric-type, intestinal-type, and pancreatobiliary-type. Some studies suggest that these subtypes are associated with specific molecular pathways and with different risks of malignant progression and different prognosis, making them an interesting feature for risk stratification. Generally, gastric-type IPMNs are low-grade and associated with the smallest likelihood of invasion, pancreatobiliary-type IPMNs are high-grade and associated with the highest likelihood of invasion, and intestinal-type IPMNs are high-grade and associated with mucinous "colloid" adenocarcinomas (see Fig. 1) [1, 2]. However, subtyping of IPMNs is often not so clear cut as different subtypes can be mixed in one IPMN and high interobserver variability is known [3]. Moreover, it has been suggested that gastric- and pancreatobiliary-type IPMNs are the same entity but with different grades of dysplasia (see Fig. 1) [4]. Omori et al. studied 60 cases of intestinal-type IPMN in an effort to elucidate the molecular mechanisms of the

Lodewijk A. A. Brosens

1.a.a.brosens@umcutrecht.nl

1 Department of Oncology, Johns Hopkins University, Baltimore, USA

2 Department of Pathology, University Medical Center Utrecht, Utrecht University, Heidelberglaan 100, 3584 Utrecht, CX, Netherlands development and the progression of these IPMNs from lowgrade to high-grade dysplasia.

The study by Omori et al. shows interesting dynamics in the development of IPMNs and pancreatic cancers. Based on their observations in tissue slides, they hypothesize the transition from gastric-type IPMN to intestinal-type IPMN. Although the claim to see evolutionary dynamics in tissue slides, fixed in time, is subjective, the authors have tried to substantiate their claims with auxiliary techniques.

Historically, pathologists have used (immuno)histochemistry to investigate the common expression of certain molecules to infer a (clonal) relationship between cells, based on the heritability of epigenetic programs. Omori et al. describe shared expression of CDX2 in both gastric-type IPMN and intestinal-type IPMN. This observation provides evidence for a clonal relationship between these lesions.

Omori et al. did an attempt to further substantiate the morphologically assumed clonal relationships between gastrictype and intestinal-type IPMN by mutation analysis of commonly mutated driver genes (GNAS and KRAS). Importantly, shared mutations in genes like GNAS and KRAS provide only limited evidence of clonal relatedness, as the same hotspot mutation in these genes can easily be seen in unrelated lesions through two different molecular events. In contrast, multiple shared passenger mutations (e.g., synonymous mutations) that do not influence the fitness of a clone, or mutations in driver

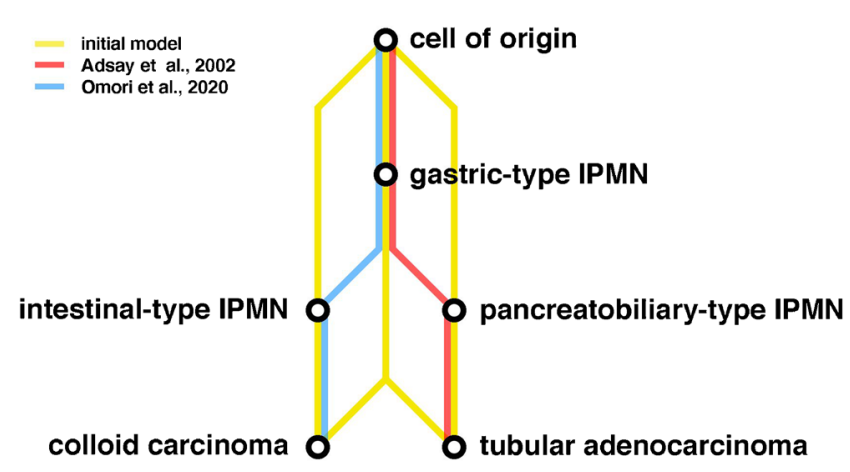

Fig. 1 Schematic figure of the different proposed evolutionary trajectories in IPMN 
genes without mutational hotspots, provide stronger evidence for clonal relatedness between two samples. The probability that two separate molecular events result in the same mutation and grow out to detectable variant allele frequencies (VAFs) is extremely small.

Although not adding to the evidence of clonal relatedness, Omori et al. also showed presence of RNF43 mutations in 8/ 14 intestinal-type IPMNs, which were not present in adjacent gastric-type IPMN. Assuming that gastric-type IPMN and intestinal-type IPMN are indeed clonally related, accumulation of these extra mutations can be interpreted as further evolution of these lesions and thus directionality. RNF43 is an ubiquitin ligase that suppresses $\mathrm{Wnt} / \beta$-catenin signaling. The unopposed Wnt pathway in intestinal-type IPMNs is also illustrated by aberrant expression of $\beta$-catenin in $80 \%$ of the intestinal-type IPMNs.

As fashionable in current scientific literature, Omori et al. connected the different observations in a coherent story. CDX2 is presented as the protagonist in the transition from gastric-type IPMN to intestinal-type IPMN. The expression of this marker is described to precede the expression of MUC2, another intestinal marker. Furthermore, Omori et al. show that CDX2 increases both the expression of p21, an inhibitor of cell proliferation, and cell proliferation itself (indicated by Ki67) albeit in different cellular compartments, suggesting a pivotal role for these events in the acquisition of the intestinal phenotype at the morphological level. The relationship between CDX2 and p21 has been described in the literature: p21 is a transcriptional target of CDX2 [5]. However, several important questions remain unanswered, such as the mechanism of CDX2 activation during progression and how CDX2 promotes cell proliferation, since evidence coming from studies in colon cancer rather suggests a tumor-suppressing role of CDX2 [6].

A more general remark to the proposed theory of Omori et al. is that the de novo expression of multiple celldifferentiation markers during tumor progression is an oddity elsewhere in the body. During neoplastic progression, clones accumulate somatic mutations and most mutations are deleterious. This phenomenon continues in cancers and is often mentioned as the reason for "dedifferentiation" and loss of differentiation markers. However, de novo gain of expression of different markers can be seen in metaplasia. An exact copy of this phenomenon is seen in the stomach, where gastric epithelium can show intestinal metaplasia during its progression to gastric cancer. Additionally, CDX2 has also been implicated in intestinal metaplasia of the stomach [7-9].

In summary, Omori et al. combined (immuno)histochemistry and molecular analyses to investigate the molecular mechanisms during progression of IPMNs. They provide for the first time some evidence that gastric-type and intestinal-type IPMN are clonally related and that CDX2 is an early marker of transformation from gastric-type IPMN to intestinal-type IPMN. Further studies are necessary to definitively confirm the clonal relationship and the evolutionary directionality between gastric-type and intestinal-type IPMNs and address mechanisms behind CDX2 activation.

If a critical amount of evidence eventually shows the proposed clonal relationship between gastric-type, intestinaltype, and, possibly, pancreatobiliary-type IPMN, a nomenclature update will be warranted, since the current naming does not infer a clonal relationship. Gastric-type IPMN is already known to be associated with low-grade dysplasia, while intestinal-type and pancreatobiliary-type IPMN are associated with high-grade dysplasia. Changing to a nomenclature, which infers a clonal relationship and is based on the grade of dysplasia, might be more informative about the risk for malignant transformation.

Author's contributions Dr. Noë and Dr. Brosens both designed and wrote the manuscript.

Funding The Dutch Digestive Foundation grant MLDS CDG 14-020 (LAAB)

\section{Compliance with ethical standards}

Conflict of interest The authors declare that they have no conflict of interest.

\section{References}

1. Tan MC, Basturk O, Brannon AR et al (2015) GNAS and KRAS mutations define separate progression pathways in intraductal papillary mucinous neoplasm-associated carcinoma. J Am Coll Surg 220: 845-854.e1

2. Koh YX, Zheng HL, Chok A-Y, Tan CS, Wyone W, Lim TKH, Tan DMY, Goh BKP (2015) Systematic review and meta-analysis of the spectrum and outcomes of different histologic subtypes of noninvasive and invasive intraductal papillary mucinous neoplasms. Surgery 157:496-509

3. Schaberg KB, DiMaio MA, Longacre TA (2016) Intraductal papillary mucinous neoplasms often contain epithelium from multiple subtypes and/or are unclassifiable. Am J Surg Pathol 40:44-50

4. Adsay NV, Conlon KC, Zee SY, Brennan MF, Klimstra DS (2002) Intraductal papillary-mucinous neoplasms of the pancreas: an analysis of in situ and invasive carcinomas in 28 patients. Cancer 94:6277

5. Bai Y-Q, Miyake S, Iwai T, Yuasa Y (2003) CDX2, a homeobox transcription factor, upregulates transcription of the p21/WAF1/CIP1 gene. Oncogene 22:7942-7949

6. Chawengsaksophak K, James R, Hammond VE, Köntgen F, Beck F (1997) Homeosis and intestinal tumours in Cdx2 mutant mice. Nature 386:84-87 
7. Bai Y-Q, Yamamoto H, Akiyama Y, Tanaka H, Takizawa T, Koike M, Kenji Yagi O, Saitoh K, Takeshita K, Iwai T, Yuasa Y (2002) Ectopic expression of homeodomain protein CDX2 in intestinal metaplasia and carcinomas of the stomach. Cancer Lett 176:47-55

8. Mutoh H, Hakamata Y, Sato K, Eda A, Yanaka I, Honda S, Osawa H, Kaneko Y, Sugano K (2002) Conversion of gastric mucosa to intestinal metaplasia in $\mathrm{Cdx} 2$-expressing transgenic mice. Biochem Biophys Res Commun 294:470-479
9. Silberg DG, Sullivan J, Kang E, Swain GP, Moffett J, Sund NJ, Sackett SD, Kaestner KH (2002) Cdx2 ectopic expression induces gastric intestinal metaplasia in transgenic mice. Gastroenterology 122:689-696

Publisher's note Springer Nature remains neutral with regard to jurisdictional claims in published maps and institutional affiliations. 